\title{
Study of local reconnection physics in a laboratory plasma
}

\author{
Hantao Ji, Troy Carter, Scott Hsu, and Masaaki Yamada \\ Princeton Plasma Physics Laboratory, Princeton University, New Jersey 08543, U.S.A.
}

(Received June 20, 2000; Revised January 25, 2001; Accepted February 28, 2001)

\begin{abstract}
A short review of physics results obtained in the Magnetic Reconnection Experiment (MRX) is given with an emphasis on the local features of magnetic reconnection in a controlled environment. Stable two-dimensional (2D) current sheets are formed and sustained by induction using two internal coils. The observed reconnection rates are found to be quantitatively consistent with a generalized Sweet-Parker model which incorporates compressibility, unbalanced upstream-downstream pressure, and the effective resistivity. The latter is significantly enhanced over its classical values in the low collisionality regime. Strong local ion heating is measured by an optical probe during the reconnection process, and at least half of the increased ion energy must be due to nonclassical processes, consistent with the resistivity enhancement. Characteristics of high frequency electrostatic and electromagnetic fluctuations detected in the current sheet suggest presence of the lower-hybrid-drift like waves with significant magnetic components. The detailed structures of current sheet are measured and compared with Harris theory and two-fluid theory.
\end{abstract}

\section{Introduction}

Magnetic reconnection plays a crucial role in determining the topology of magnetic fields in space and laboratory plasmas (Vasyliunas, 1975; Biskamp, 1994). Although this is a localized process, it often causes fundamental changes in macroscopic configurations, such as in solar flares, magnetospheric substorms, and relaxation processes in laboratory plasmas. Magnetic reconnection also provides the most plausible mechanism for releasing the energy stored in the magnetic field to plasma kinetic and thermal energies as observed in solar flares, auroral phenomena, and laboratory plasmas. Over more than fifty years of its history, study of magnetic reconnection has been largely theoretical as represented by the Sweet-Parker model (Parker, 1957; Sweet, 1958) and the Petschek model (Petschek, 1964). Recently, availability of computer simulation as a research tool has brought about an explosive amount of literature on numerical studies of magnetic reconnection physics in great detail.

In comparison with these theoretical and numerical efforts, laboratory investigations of magnetic reconnection have been relatively rare (Yamada, 1999). The first detailed experiment (Stenzel and Gekelman, 1981) on magnetic reconnection was carried out in linear geometry and in the electron magnetohydrodynamic (EMHD) regime, in which ions are unmagnetized. Fluctuations were measured in the current sheet region in detail. More recently, the global nature of magnetic reconnection in the MHD regime, where both ions and electrons are magnetized, has been studied in the TS-3 device (Yamada et al., 1990; Ono et al., 1996). Effects of the third (toroidal) component of magnetic field and global ion heating have been reported. Since 1995, a

Copy right (c) The Society of Geomagnetism and Earth, Planetary and Space Sciences (SGEPSS); The Seismological Society of Japan; The Volcanological Society of Japan; The Geodetic Society of Japan; The Japanese Society for Planetary Sciences. series of extensive experiments has been performed in the Magnetic Reconnection Experiment or MRX (Yamada et al., 1997b) to comprehensively study magnetic reconnection from both local and global points of view in a controlled MHD regime, where both location and timing of reconnection process are specified. This short article is intended to summarize the experimental results obtained recently in MRX with an emphasis on the local features at the diffusion region where ions become unmagnetized, in contrast to the outer MHD regions.

\section{Experimental Apparatus and Diagnostics}

In MRX, the plasma is formed and controlled by a pair of internal coils (called flux cores, with a $37.5 \mathrm{~cm}$ major radius and $\mathrm{a} \simeq 10 \mathrm{~cm}$ minor radius), which creates a quadrupole field configuration. The magnetic domain can be divided into three sections: two private sections surrounding each flux core and one public section surrounding both flux cores, which are separated from each other by $50 \mathrm{~cm}$ surface to surface (see Fig. 1(a)). When poloidal flux in the private sections (or toroidal currents in the flux cores) is reduced, the poloidal flux is "pulled" back from the public section to the private sections resulting in magnetic reconnection as illustrated in Fig. 1(b). In addition to the poloidal field shown in Fig. 1, a toroidal field can be removed (added) at the diffusion region resulting in null-helicity (co-helicity) reconnection. The toroidally symmetric shape of the flux cores ensures global 2D geometry for magnetic reconnection. Note the use of cylindrical coordinates $(R, \theta, Z)$ as defined in Fig. 1(a).

The low temperature $(<50 \mathrm{eV})$ and short-pulsed $(<1 \mathrm{~ms})$ MRX plasmas have the advantage that internal probes can be used routinely (Yamada et al., 1997b). Triple Langmuir probes (Ji et al., 1991) are used to measure electron density $\left(n_{e}\right)$ and temperature $\left(T_{e}\right)$ simultaneously. The plasma 
(a) Before reconnection

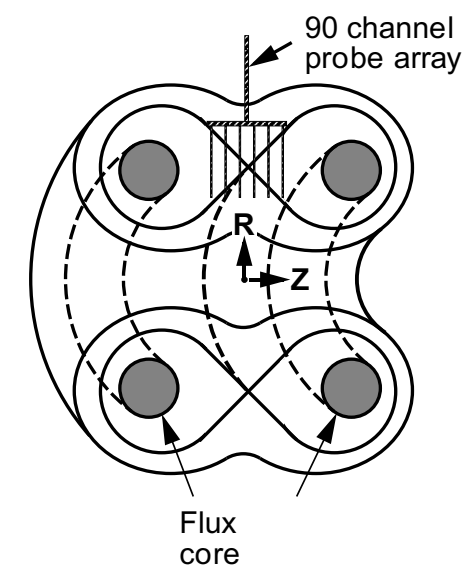

(b) "Pull" reconnection

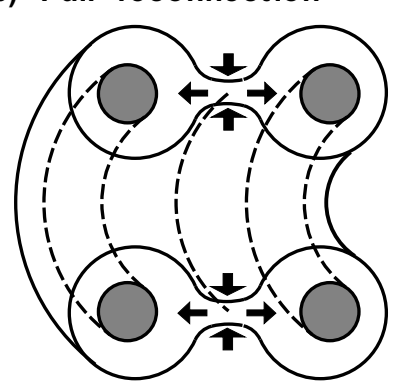

Fig. 1. An illustration of the MRX geometry for magnetic reconnection: (a) a quadrupole configuration formed by two flux cores, providing one public and two private regions, (b) two-dimensional magnetic reconnection induced by pulling flux from the public back to the private regions.

density measurement has been calibrated by a laser interferometer which measures the line-integrated density. All three components of $\mathbf{B}$ are measured during the reconnection process by a 90 channel 2D pick-up coil array with 4 $\mathrm{cm}$ resolution around the diffusion region (see Fig. 1(a)). Another 60 channel 2D pick-up coil array with $6 \mathrm{~cm}$ resolution in the $R$ direction and $8 \mathrm{~cm}$ resolution in the $Z$ direction is also used to measure magnetic profiles at the smaller radii where magnetic field is smoother. The poloidal flux function can be obtained by integration of $B_{Z}$ over the radius, $\Psi(R, Z)=2 \pi \int_{0}^{R} B_{Z}(R, Z) R d R$. A finer $1 \mathrm{D}$ pick-up probe array with $0.5 \mathrm{~cm}$ resolution is used to measure the $B_{Z}$ profile across the current sheet. Local flow velocity in $R$ and $Z$ directions can be determined either by a Mach probe or time evolution of $\Psi(R, Z)$, i.e., $V_{X}=-(\partial \Psi / \partial t) /(\partial \Psi / \partial X)$ ( $X=R$ in the upstream region and $X=Z$ in the downstream region). The latter method is valid when the resistive effects are negligible, a condition satisfied outside the diffusion region. Results from both methods are in good agreement, and the latter has been used routinely because of its convenience. Probe perturbation of the plasma is estimated quantitatively and observed to be less than 5\% of the measured magnetic fields (Yamada et al., 1997a).

In order to determine ion flow and temperature, an optical probe called the Ion Dynamics Spectroscopy Probe or IDSP (Fiksel et al., 1998) was inserted to collect plasma light from a localized $(\sim 5 \mathrm{~cm})$ volume through fiber optics to a $1 \mathrm{~m}$ monochromator imaged with a CCD camera. Two perpendicular lines-of-sight can give simultaneous Doppler broadening and relative Doppler shift information, thus providing measurements of $R$ and $Z$ components of the velocity vector and temperature. The brightness of plasma emission limits the time resolution to about $10 \mu \mathrm{s}$. Typical plasma parameters are as follows: $B<0.5 \mathrm{kG}, T=5-30 \mathrm{eV}$, and $n_{e}=0.2-1.5 \times 10^{20} \mathrm{~m}^{-3}$.

\section{Experimental Realization of Sweet-Parker-Like Magnetic Reconnection in Two Dimensions}

The most fundamental MHD model of magnetic reconnection in two dimensions was given by Sweet (1958) and Parker (1957). A key element of this model is the existence of a 2D "diffusion region"- essentially a rectangular box where the magnetic field diffuses and reconnects. The dimensions of such a "box" are of crucial importance since it essentially decides the rate of magnetic reconnection by balancing incoming and outgoing plasma and flux flows and thus the time scale for reconnection. The length of this box is of macroscopic scale but its width is determined by the local plasma resistivity which causes magnetic diffusion. The Sweet-Parker model uses resistivities estimated by classical theories, such as the Spitzer resistivity (Spitzer, 1962). Under the assumptions of steady state, imcompressibility, and uniform plasma pressure outside the diffusion region, an important prediction by the Sweet-Parker model is a normalized reconnection rate of,

$$
\frac{V_{R}}{V_{A}}=\sqrt{\frac{\eta}{\mu_{0} L V_{A}}} \equiv \frac{1}{\sqrt{S}},
$$

where $V_{A}$ is Alfvén speed defined by reconnecting magnetic field, $\eta$ is the plasma resistivity, $L$ is the length of diffusion region, and $S$ is called Lundquist number.

Despite its importance and long history, the Sweet-Parker model had never been tested either in laboratory or in space. The first quantitative tests were possible in MRX since all essential plasma parameters were measured during magnetic reconnection by an extensive set of diagnostics described in Section 2. First of all, it should be emphasized that, in qualitative agreement with Sweet-Parker model, a robustly stable two-dimensional diffusion region (or current sheet) is formed and sustained during the "pull" reconnection regardless of the existence of the third component (toroidal field, $B_{\theta}$ ) as long as the reconnection is driven. (Indeed, it would not be surprising if the current sheet disrupts especially during the null-helicity reconnection.) Two examples are shown in Fig. 2, where the measured contours of the poloidal flux before and during the reconnection are plotted for both nullhelicity and co-helicity cases. Although the detailed structures differ between these two cases (Yamada et al., 1997a), a common feature is that the formed current sheet is robustly stable (lifetime $>20$ Alfvén time) and globally axisymmetric as long as reconnection is being driven.

Furthermore, detailed quantitative comparisons have been made between the measured reconnection rates and the predictions by the Sweet-Parker model (Ji et al., 1998). It turned out that the measured reconnection rates do not agree with the simple prediction of Eq. (1), because several assumptions made by the original Sweet-Parker model do not hold true in MRX. First, the plasma is compressible, i.e., 
(a) Null-helicity reconnection (b) Co-helicity reconnection
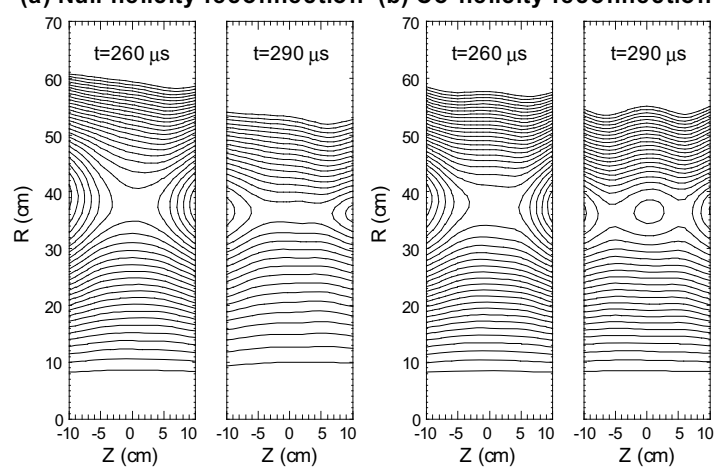

Fig. 2. Time evolution of contours of poloidal flux measured by internal magnetic probe arrays in the (a) null-helicity case and (b) co-helicity case, respectively.

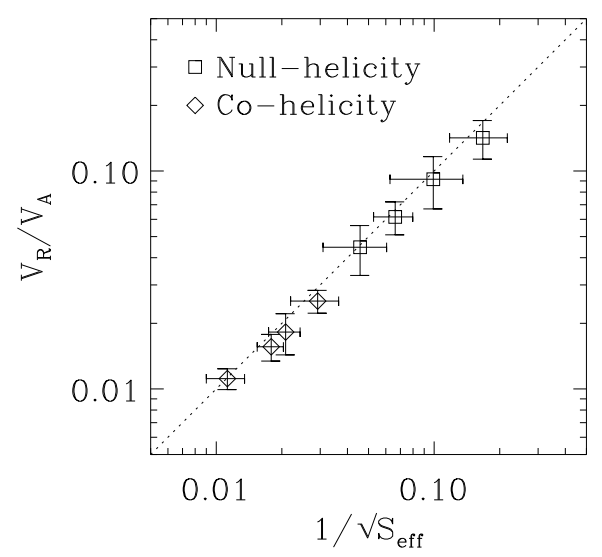

Fig. 3. Comparison of the measured reconnection rates to the predictions by a generalized Sweet-Parker model for both null-helicity and co-helicity cases.

$\dot{n} \neq 0$, especially when the magnetic field is strong or the Lundquist number is large. Compressibility accelerates reconnection transiently. Second, the plasma pressure outside the diffusion region is not uniform, namely, downstream pressure $p_{\text {down }}$ becomes larger than upstream pressure $p_{\text {up }}$ by as much as a factor of two or more. Much higher $p_{\text {down }}$ slows outflow to only 5-30\% of Alfvén speed, resulting in slower reconnection rates. Lastly and most importantly, the effective plasma resistivity $\eta^{*}$ becomes larger than the classical resistivity by as much as an order of magnitude in the low collisionality regimes, greatly accelerating the reconnection process.

By correcting these assumptions, the Sweet-Parker model can be generalized (Ji et al., 1998) by defining an effective Lundquist number,

$$
S_{\text {eff }}=\frac{\mu_{0} L V_{A}}{\eta^{*}} \cdot \frac{1}{1+L \dot{n} / n V_{Z}} \cdot \frac{V_{Z}}{V_{A}}
$$

where the outflow $V_{Z}$ is determined by

$$
V_{Z}^{2}=V_{A}^{2}(1+\kappa)-2\left(p_{\text {down }}-p_{\text {up }}\right) / \rho .
$$

Here $\kappa$ represents a small correction due to the downstream tension force (Ji et al., 1999). Figure 3 shows satisfactory agreement spanning over a decade in magnitude between the experiments and the generalized Sweet-Parker model for both null-helicity and co-helicity reconnection. It should be pointed out that the much slower reconnection rates in the co-helicity cases compared to the null-helicity cases are due to a combined effect of lower compressibility, larger $p_{\text {down }}-p_{\text {up }}$, and less enhancement of the effective resistivity.

\section{Resistivity Enhancement and Measurements of Fast Fluctuations}

As seen in the last section, the most important deviation of the reconnection process in MRX from the classical SweetParker model is the enhancement of the effective plasma resistivity over its classical values. It has been found that the resistivity enhancement strongly depends on density or equivalently the collisionality (Ji et al., 1998), as shown in Fig. 4. The enhancement is largest in the low collisionality regime where the mean free path of particles $(>10 \mathrm{~cm})$ is much longer than the current sheet thickness $(<2.5 \mathrm{~cm})$ or the ion skin depth, an important scale length in the perpendicular direction of magnetic field. Collisionless effects, such as two-fluid effects and ion kinetic effects, can be more important than Coulomb collisions. Indeed, plenty of free energy, as a form of large relative drift $V_{d}\left(\equiv j_{\theta} / e n\right)$ between ions and electrons, exists at the diffusion region in the low density cases, likely resulting in current-driven microinstabilities (see, e.g., Huba et al., 1977). Experimentally, the effective resistivity is determined (Ji et al., 1998) by $\eta^{*}=E_{\theta} / j_{\theta}=\mu_{0} E_{\theta} \delta / B_{Z}$, where $E_{\theta}$ is the toroidal electric field determined by time derivative of poloidal flux, $-\partial \Psi / \partial t$, and $\delta$ is the thickness of the current sheet. Therefore, the enhancement in resistivity is equivalent to a larger current sheet thickness than predicted by the classical resistivity. Indeed, the measurements show that $\delta$ is determined by ion skin depth (Yamada et al., 2000; also see Section 6), a fact which can be translated to a constant drift parameter, $V_{d} / V_{\text {thi }}\left(V_{\text {thi }}=\right.$ ion thermal velocity $)$, over a wide range in density (Ji et al., 1999). When the density is reduced, the drift parameter needs to be increased to keep a constant current density thus a constant effective resistivity. Therefore, the observation of a constant drift parameter suggests that current-driven instabilities can provide a mechanism to

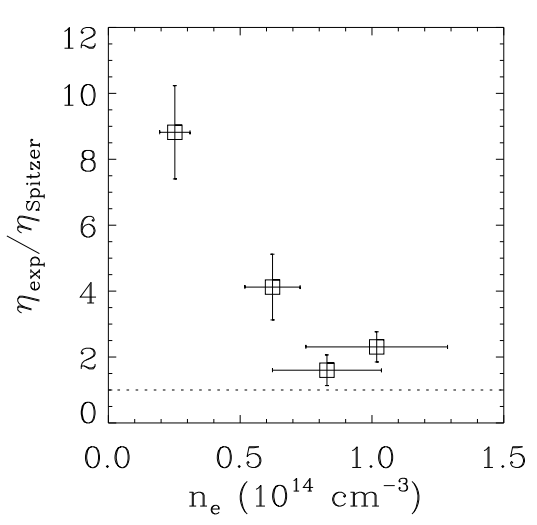

Fig. 4. The measured resistivity enhancement as a function of plasma density during null-helicity reconnection. 
limit current density, thus controlling the reconnection rate.

Experimental efforts have begun in MRX to detect fast fluctuations at the current sheet with a frequency response up to as high as $100 \mathrm{MHz}$, which is well above the lowerhybrid frequency. Both electrostatic and magnetic fluctuations have been successfully identified and measured (Carter et al., 2001) during reconnection, by using miniature transducers installed in the probe shaft right after Langmuir or magnetic probe tips. One example is shown in Fig. 5, where both electrostatic and electromagnetic fluctuations appear in the current sheet after the current sheet is formed and the reconnection is driven. Radial scans of fluctuation measurements indicate that both electrostatic and electromagnetic fluctuations peak around the current sheet. The measured frequency spectra, as shown also in Fig. 5, indicate that most fluctuations are in the frequency range between the ion cyclotron and lower hybrid frequencies. Measurements of wavenumber spectra using multiple probes show that the phase velocities of these fluctuations are qualitatively consistent with the relative drift velocity, pointing to lower-hybrid-drift like waves with significant magnetic components (Carter et al., 2001). Understanding the nature of these fluctuations represents an important step in the research of magnetic reconnection.
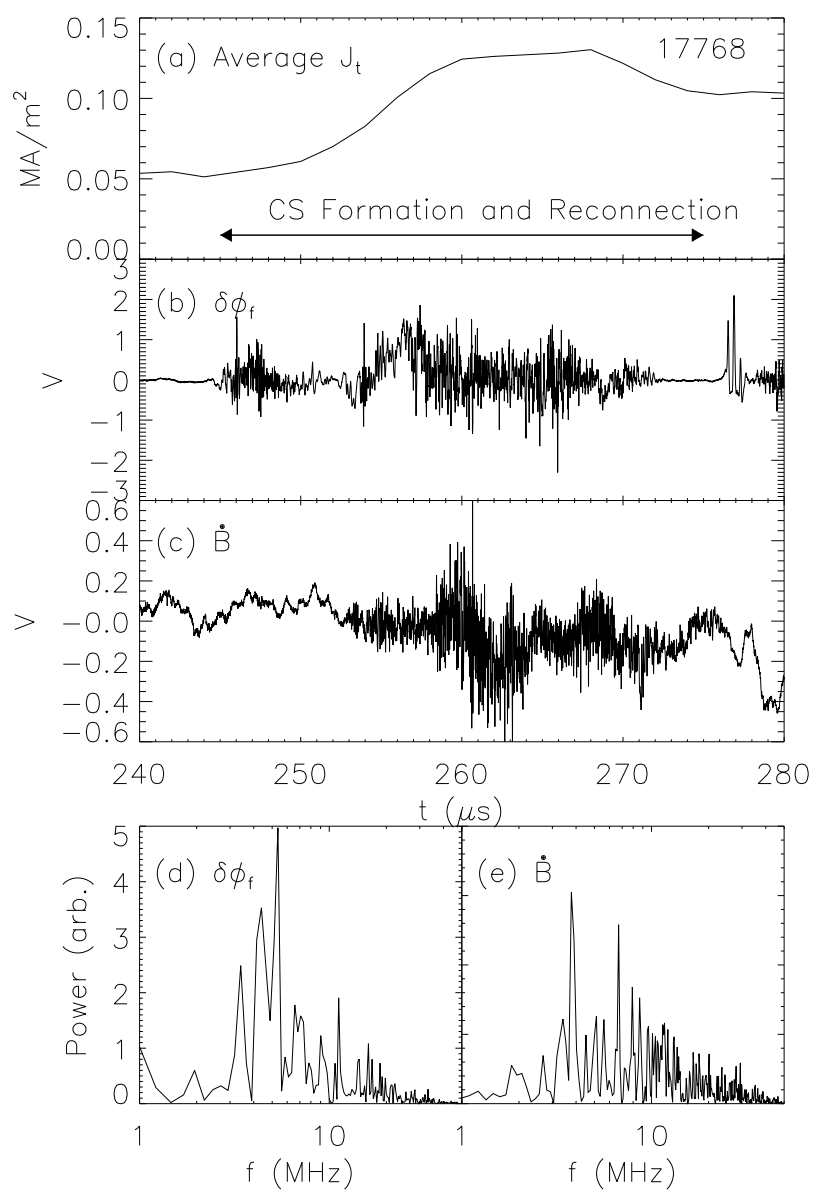

Fig. 5. Examples of the measured electrostatic (b) and electromagnetic (c) fluctuations as the current sheet (a) is formed and null-reconnection is driven. Frequency spectra of both signals in the range of of lower-hybrid frequency are also shown ((d) and (e)).

\section{Measurements of Nonclassical Ion Heating}

Another important aspect of magnetic reconnection is its ability to convert magnetic energy to plasma energy, obserable as plasma or particularly ion heating. High temperatures observed in solar corona (Priest, 1998), magnetospheric plasmas (Dungey, 1961), and laboratory plasmas (Ejiri and Miyamoto, 1995) have been attributed to magnetic reconnection. Electron heating was observed during reconnection in the EMHD regime (Stenzel et al., 1982), and global ion heating was observed in the TS-3 experiments and was attributed to thermalization of Alfvénic flows (Ono et al., 1996). Alfvénic ion jets were also measured in the SSX experiment when two spheromaks interact with each other (Kornack et al., 1998). However, there have been no direct demonstrations of ion heating localized at a well-characterized diffusion region during magnetic reconnection.

Local ion temperatures have been successfully measured (Hsu et al., 2000) in MRX by the IDSP probe (Fiksel et al., 1998) and a $1 \mathrm{~m}$ monochromator imaged with a CCD camera. Majority ion temperature and flow is accurately determined thanks to the high resolution of pixels of the CCD camera ( $\sim 20$ pixels within the broadened He II line centered at $468.6 \mathrm{~nm}$ ). Figure 6 shows the time evolution of the temperature of helium ions at the center of the diffusion region for both null-helicity and co-helicity reconnection. Ion temperature for the case without reconnection in the nullhelicity configuration is also shown for reference. The $T_{i}$

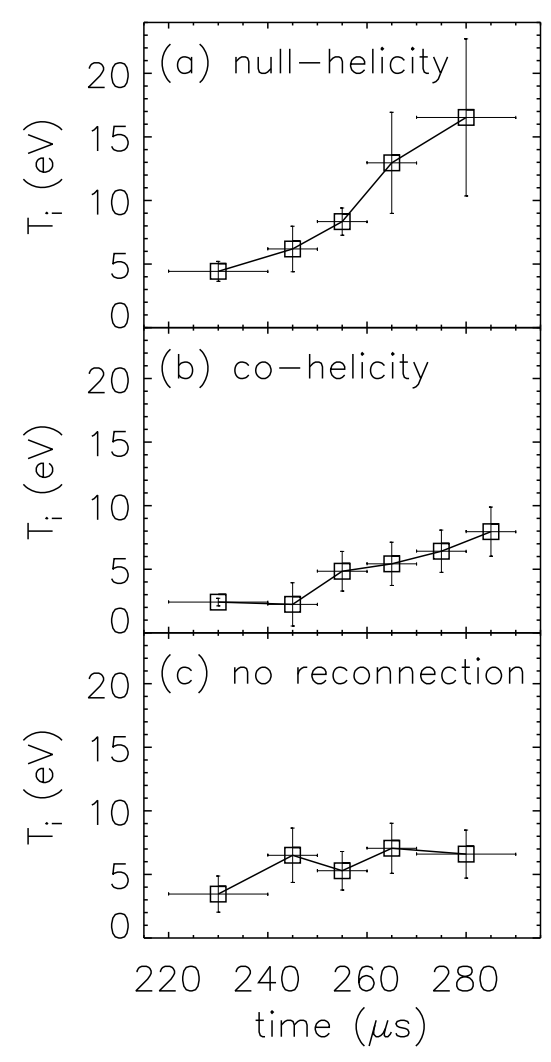

Fig. 6. Time evolution of the measured helium ion temperature at the center of the diffusion region for both null-helicity and co-helicity reconnection, and for the case without reconnection in a null-helicity setup. 
rises by more than a factor of three for the null-helicity case and by a factor of two for the co-helicity case when reconnection is driven while there are almost no changes when no reconnection is driven. We note that less dramatic ion heating in the co-helicity case is consistent with its slower reconnection rates ( $\mathrm{Ji}$ et al., 1999). As a more convincing evidence of direct ion heating due to reconnection, Fig. 7 shows radial profile of $T_{i}$ increase and reconnecting magnetic field. Ions are heated only at the diffusion region during the reconnection.

An immediate question arises regarding whether the observed ion heating can be accounted for by the know classical processes. A thorough estimate (Hsu et al., 2001) using Braginskii's formula (1965) has been performed taking into account all possible effects on energy gains (by electrons, compression, and viscosity) and energy losses (by neutrals, convection, and conduction). All these processes can only explain approximately half of the ion energy increase, leaving another half unaccounted. This must have been converted to ion energy nonclassically.

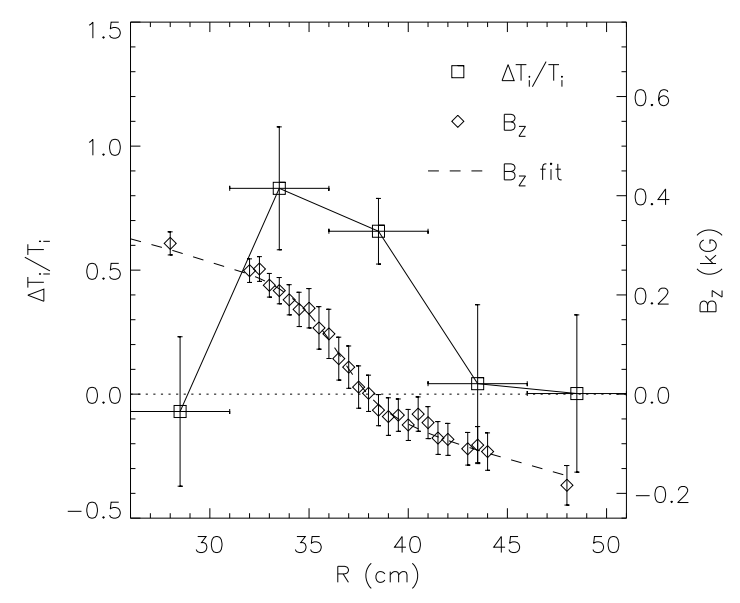

Fig. 7. Radial profile of the relative rise of $T_{i}$ during null-helicity reconnection and reconnecting magnetic field averaged over the same time.

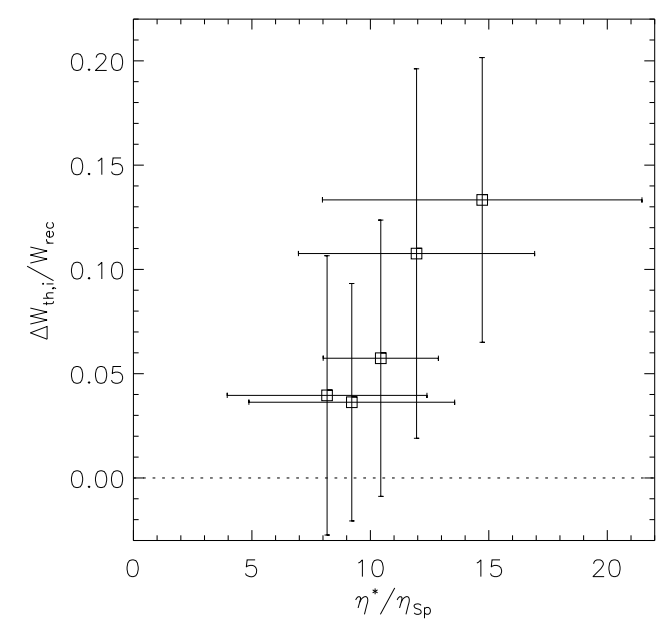

Fig. 8. Increase in ion energy normalized by reconnected magnetic energy as a function of the resistivity enhancement, $\eta^{*} / \eta_{\mathrm{Sp}}$.
A logical next question arises on how this nonclassical ion heating is related to the observed resistivity enhancement. Indeed, the increase in ion energy normalized by reconnected magnetic energy increases with the enhancement factor, as shown in Fig. 8. This suggests that the same (nonclassical) mechanism(s) for resistivity enhancement also directly heat the ions in MRX.

\section{Study of Current Sheet Structure}

Structures of the current sheet hold significant clues about the physical mechanisms which control reconnection. Magnetic profiles have been measured by a 1D pick-up coil array with resolution of $0.5 \mathrm{~cm}$ (Yamada et al., 1997b, 2000). It was found that the reconnecting field profile fits well with a Harris-type profile (Harris, 1962), $B(x) \sim \tanh (x / \delta)$. Fitting to error functions, as in MHD simulations (Biskamp, 1986), gives slightly worse $\chi^{2}$, although there are no significant statistical advantages over fitting to arctangent, as shown in Fig. 9. Since the original Harris solution assumes $T_{e}=T_{i}$, which does not hold true in MRX, the solution of the Vlasov equation has been generalized to include $T_{e} \neq T_{i}$ cases (Yamada et al., 2000) to predict the thickness

$$
\delta=\frac{c}{\omega_{\mathrm{pi}}} \frac{\sqrt{2} V_{s}}{V_{d}},
$$

where $V_{s} \equiv \sqrt{\left(T_{e}+T_{i}\right) / m_{i}}$. This relation has been verified experimentally (Yamada et al., 2000).

In order to study both magnetic and electric structures of the current sheet in MRX, further generalizations are needed to include nonuniform electron and ion flows. In the full Vlasov equation, this cannot be done without introducing other exotic effects, such anisotropic temperatures (Mahajan and Hazeltine, 2000). However, a simple two-fluid theory can be constructed (Ji et al., 2000) to give a nonlinear equation for the magnetic field in a normalized form,

$$
\frac{\partial B}{\partial x}=-\frac{V}{\sqrt{2}}\left(1-B^{2}\right),
$$

where $x$ and $B$ are normalized by $c / \omega_{\text {pi }}$ and $\sqrt{2 \mu_{0} n_{0}\left(T_{e}+T_{i}\right)}$, respectively, and $V \equiv V_{d} / V_{s}$. When $T_{e}+T_{i}$ is a constant, as is mostly the case in MRX, the predicted magnetic and electric profiles are

$$
\begin{aligned}
E_{x} & =-\frac{T_{e} V_{i}+T_{i} V_{e}}{T_{e}+T_{i}} B_{z} \\
B_{z} & =B_{0} \tanh \left(\int_{0}^{x} \frac{V}{\sqrt{2}} d x\right),
\end{aligned}
$$

where $V_{i}\left(V_{e}\right)$ is ion (electron) flow in the current $(y)$ direction. Because $V$ resides within the integration before entering as the argument of hyperbolic tangent, the $B$ profile is not sensitive to the details of $V$, and it is always very close to the shape of tanh as long as $V$ is a reasonably smooth function of $x$. Three examples are given in Fig. 10, which shows that despite substantial changes in the $V$ profiles, the corresponding $B$ profiles fit well to tanh function by adjusting amplitude and thickness, demonstrating the robustness of Harris-like profiles of magnetic field. Detailed measurements of the structures of electric field and flows are underway; they will be compared to the two-fluid theory in 


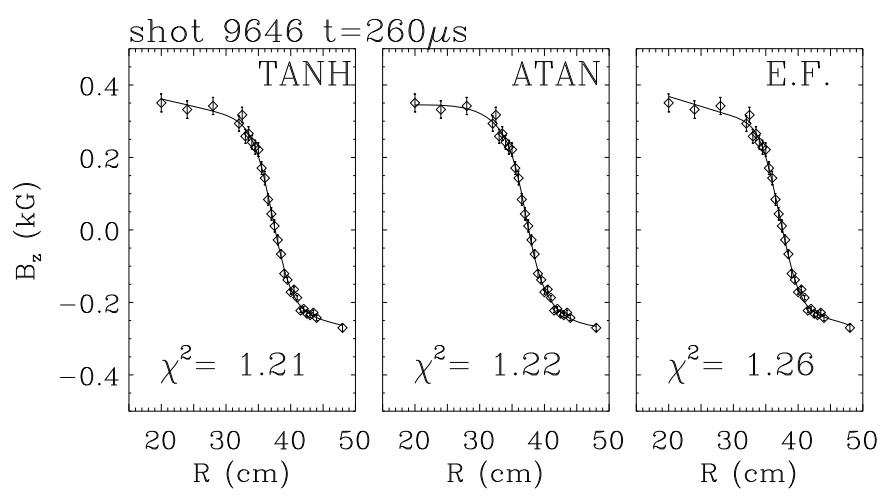

Fig. 9. Measured magnetic profile and its fittings to hyperbolic tangent, arctangent, and error function.

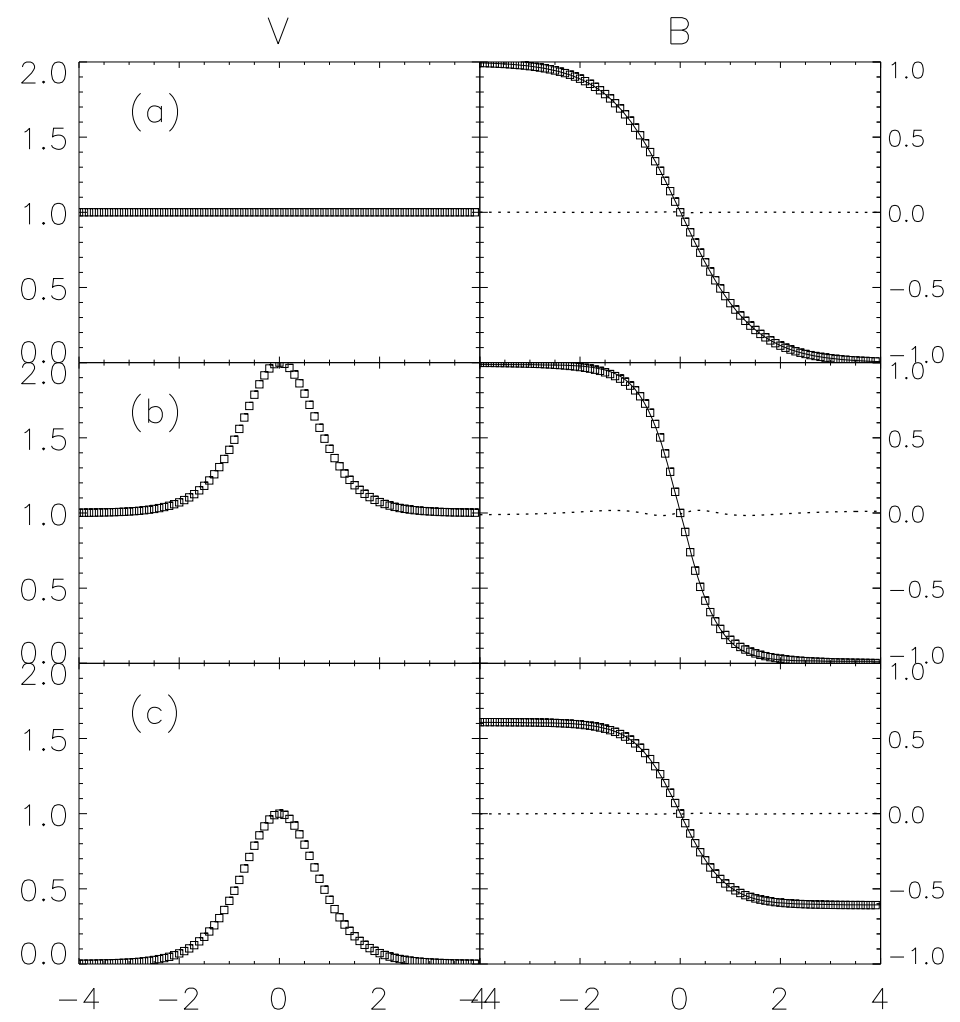

Fig. 10. Three examples to illustrate robustness of the hyperbolic tangent shape of the magnetic field. Profiles of $V$ (left) and $B$ (right) are plotted as functions of $x$ for cases of a constant $v(\mathrm{a})$, doubled $V$ around $x=0(\mathrm{~b})$, and a peaked $V$ around $x=0$ (c). Squares indicate solutions of Eq. (5). The solid lines and dotted lines are fitted tanh curves and deviations from tanh, respectively.

order to understand non-MHD effects in relation with the observed fast reconnection and nonclassical ion heating.

\section{Conclusions}

A brief review is given on the local physics results obtained in MRX. Overall, the observed magnetic reconnection is qualitatively and quantitatively consistent with the two-dimensional MHD model given by Sweet and Parker. Qualitatively, stable two-dimensional current sheets are formed and sustained as long as the reconnection is driven. Quantitatively, the observed reconnection rates are also consistent with the Sweet-Parker model, but only after it is generalized to incorporates compressibility, unbalanced upstream-downstream pressure, and the effective resistivity.
The latter is significantly enhanced over its classical values in the low collisionality regimes. Strong local ion heating is measured by an optical probe during the reconnection process, and at least half of the increased ion energy must be due to nonclassical processes, consistent with the observed resistivity enhancement. Characteristics of high frequency electrostatic and electromagnetic fluctuations detected in the current sheet suggest presence of the lowerhybrid-drift like waves with significant magnetic components. The detailed structures of the current sheet are measured and compared with Harris' theory and two-fluid theory, which explains why the hyperbolic tangent shape of the reconnecting magnetic field is preferred. In addition, a novel optical system using Laser Induced Fluorescense 
(Trintchouk et al., 2000) is now operational, allowing noninvasive study of the local reconnection physics, such as density structures and ion heating.

Acknowledgments. The author is grateful to Drs. F. Trintchouk and R. Kulsrud for their collaborations.

\section{References}

Biskamp, D., Magnetic reconnection via current sheets, Phys. Fluids, 29, 1520-1531, 1986.

Biskamp, D., Magnetic reconnection, Physics Reports, 237, 179-247, 1994.

Braginskii, S. I., Transport processes in a plasma, in Reviews of Plasma Physics Vol. 1, edited by M. A. Leontovich, 205 pp., Consultants Bureau, New York, 1965.

Carter, T., et al., Measurements of lower-hybrid-drift turbulence in a reconnecting current sheet, Bull. Am. Phys. Soc., 45, 270, 2000; to be submitted to Phys. Rev. Lett.

Dungey, J. W., Interplanetary magnetic field and auroral zones, Phys. Rev. Lett., 6, 47-48, 1961.

Ejiri, A. and K. Miyamoto, Ion-heating model during magnetic reconnection in reversed-field pinch plasmas, Plasma Phys. Controll. Fusion, 37, 43-56, 1995.

Fiksel, G., D. J. Den Hartog, and P. W. Fontana, An optical probe for local measurements of fast plasma ion dynamics, Rev. Sci. Instr., 69, 2024 2026, 1998.

Harris, E. G., On a plasma sheath separating regions of oppositely directed magnetic field, Il Nuovo Cimento, 23, 115-121, 1962.

Hsu, S. C., et al., Local measurement of nonclassical ion heating during magnetic reconnection, Phys. Rev. Lett., 84, 3859-3862, 2000.

Hsu, S. C., et al., Experimental study of ion heating and acceleration during magnetic reconnection, Phys. Plasmas, 8, 1916-1928, 2001.

Huba, J., et al., Lower-hybrid-drift instability as a source of anomalous resistivity for magnetic-field line reconnection, Geophys. Res. Lett., 4, 125, 1977.

Ji, H., et al., Probe measurements in the REPUTE-1 reversed field pinch, Rev. Sci. Instrum., 62, 2326-2337, 1991.

$\mathrm{Ji}, \mathrm{H}$., et al., Experimental test of the Sweet-Parker model of magnetic reconnection, Phys. Rev. Lett., 80, 3256-3259, 1998.

$\mathrm{Ji}, \mathrm{H}$., et al., Magnetic reconnection with Sweet-Parker characteristics in two-dimensional laboratory plasmas, Phys. Plasmas, 6, 1743-1750, 1999.

Ji, H., et al., Study of electric and magnetic profiles of current sheet in magnetic reconnection experiment, Bull. Am. Phys. Soc., 45, 270, 2000 (to be submitted).

Kornack, T. W., P. K. Sollins, and M. R. Brown, Experimental observation of correlated magnetic reconnection and Alfvenic ion jets, Phys. Rev. E, 58, R36-R39, 1998.

Mahajan, S. M. and R. D. Hazeltine, Sheared-flow generalization of the Harris sheet, Phys. Plasmas, 7, 1287-1293, 2000.

Ono, Y., et al., Ion acceleration and direct ion heating in three-component magnetic reconnection, Phys. Rev. Lett., 76, 3328-3331, 1996.

Parker, E. N., Sweet's mechanism for merging magnetic fields in conducting fluids, J. Geophys. Res., 62, 509-520, 1957.

Petschek, H. E., Magnetic field annhilation, NASA Spec. Pub. SP-50, 425439, 1964.

Priest, E. R., et al., Nature of the heating mechanism for the diffuse solar corona, Nature, 393, 545-547, 1998.

Spitzer, L., Jr., Physics of Fully Ionized Gases, 2nd Revised edition, p. 28, Interscience Publishers, New York, 1962.

Stenzel, R. L. and W. Gekelman, Magnetic-field line reconnection experiments 1. Field topologies, J. Geophys. Res., 86, 649-658, 1981.

Stenzel, R. L., W. Gekelman, and N. Wild, Magnetic-field line reconnection experiments 4. Resistivity, Heating, and Energy-flow, J. Geophys. Res., 87, 111-117, 1982.

Sweet, P. A., The neutral point theory of solar flares, in Electromagnetic Phenomena in Cosmical Physics, edited by B. Lehnert, pp. 123-134, Cambridge University Press, New York, 1958.

Trintchouk, F., et al., Two-dimensional structure measurement in the magnetic reconnection experiment with planar laser-induced fluorescence, Bull. Am. Phys. Soc., 45, 270-271, 2000.

Vasyliunas, V. M., Theoretical models of magnetic field line merging, 1, Rev. Geophys. Space Phys., 13, 303-336, 1975.

Yamada, M., Review of controlled laboratory experiments on physics of magnetic reconnection, J. Geophys. Res., 104, 14529-14541, 1999.

Yamada, M., et al., Magnetic reconnection of plasma toroids with cohelicity and counterhelicity, Phys. Rev. Lett., 65, 721-724, 1990.

Yamada, M., et al., Identification of Y-shaped and O-shaped diffusion regions during magnetic reconnection in a laboratory plasma, Phys. Rev. Lett., 78, 3117-3120, 1997a.

Yamada, M., et al., Study of driven magnetic reconnection in a laboratory plasma, Phys. Plasmas, 4, 1936-1944, 1997b.

Yamada, M., et al., Experimental investigation of the neutral sheet profile during magnetic reconnection, Phys. Plasmas, 5, 1781-1787, 2000.

H. Ji (e-mail: hji@pppl.gov), T. Carter, S. Hsu, and M. Yamada 